\title{
Goldilocks and the NICU Part 2: Oxygen, the Necessary Evil
}

Rob Graham, R.R.T./N.R.C.P.

I dedicate this column to the late Dr. Andrew (Andy) Shennan, the founder of the perinatal program at Women's College Hospital (now at Sunnybrook Health Sciences Centre). To my teacher, my mentor and the man I owe my career as it is to, thank you. You have earned your place where there are no hospitals and no NICUs, where all the babies do is laugh and giggle and sleep.

"In utero development occurs in a relatively hypoxic environment with PaO2 of approximately $20 \mathrm{mmHg}$, (1) thus even room air represents a hyperoxic environment to the newborn infant."

In June's Neonatology Today column we discussed "just right" values relating to $\mathrm{PaCO}_{2}$. This month's focus is on oxygen.

In utero development occurs in a relatively hypoxic environment with $\mathrm{PaO}_{2}$ of approximately $20 \mathrm{mmHg}$, (1) thus even room air represents a hyperoxic environment to the newborn infant. To protect the infant from hyperoxia and resulting formation of reactive oxygen species (ROS) (free radicals), just prior to term gestation non-enzymatic antioxidants (i.e. glutathione, vitamins $C$ and $E$, and carotenoids) cross the placenta and endogenous antioxidant systems are up-regulated immediately prior to birth and are further up-regulated upon exposure to room air. Glutathione (GSH) is by far the most abundant antioxidant in the human body, and premature infants have low levels of GSH. (2)

In the premature infant the transition to the hyperoxic extra-uterine world is poorly mitigated due to several complex and interconnected factors. The transfer of antioxidants through the placenta does not take place, and there are both developmental deficits in antioxidant defenses and impairment of the ability to rapidly mount an antioxidant response when exposed to hyperoxia ${ }^{2}$. The plethora of conditions thought to be at least caused in part by ROS have been described as "Oxygen radical disease in neonatology". (3) These pathologies include chronic lung disease (CLD), intraventricular hemorrhage (IVH), retinopathy of prematurity (ROP), necrotizing enterocolitis (NEC), periventricular leukomalacia ( $P V L)$, renal damage, hemolysis ${ }^{2}$ and patent ductus arteriosis (PDA). (3)
The increased production of ROS and reactive nitrogen species (RNS) resulting from oxidative stress also alter nitric oxide (NO) signaling by reducing NO bioavailability.

ROS are a natural byproduct of metabolism (i.e. superoxide, hydrogen peroxide) and are normally quickly reduced. In the absence of antioxidant reduction (such as in the premature infant) peroxinitrite is formed from the reaction of superoxide and NO, and hydroxyl radical results from the reaction between hydrogen peroxide and iron or copper. Additional ROS can also be produced by ischemia/reperfusion, infection, hyperoxia, inflammation, free iron and Fenton reaction and mitochondrial respiratory chain. Additionally, ascorbylperoxides (AscOOH, another oxidant) are commonly present in parental nutrition and are poorly reduced in the premature infant due to low levels of GSH. Higher levels of $\mathrm{AscOOH}$ in the urine in the first week of life are associated with increased incidence of CLD and death. (2)

\section{"Traditionally, premature infants have been antioxidant supplements such as vitamin $E$ and $A$ in an effort to reduce oxidative stress based on the concept of "antioxidant imbalance". Simply administering antioxidants or their precursors may not be a silver bullet."}

Traditionally, premature infants have been antioxidant supplements such as vitamin $E$ and $A$ in an effort to reduce oxidative stress based on the concept of "antioxidant imbalance". Simply administering antioxidants or their precursors may not be a silver bullet. For instance, premature infants have been shown to be deficient in selenium, an element essential for antioxidant enzyme function. Supplementation with selenium has not been shown to reduce either CLD, ROP, or improve survival. (2)

Several other antioxidants have been studied. Cysteine is a precursor to GSH and its administration improved nitrogen balance in premature infants but also resulted in more metabolic acidosis. Similarly, n-acetylcysteine (NAC) serves as a de novo precursor to GSH production, however NAC supplementation has not shown to improve pulmonary outcomes in extremely low birth weight infants. Superoxide dismutase (SOD) has been extensively studied in the prevention of CLD, however Cochrane metanalysis failed to find sufficient evidence to support any improvement in respiratory outcomes. Of note is a post-hoc analysis that showed a

NEONATOLOGY TODAY is interested in publishing manuscripts from Neonatologists, Fellows, NNPs and those involved in caring for neonates on case studies, research results, hospital news, meeting announcements, and other pertinent topics.

Please submit your manuscript to: LomaLindaPublishingCompany@gmail.com 
reduction in ROP. ROP severity above stage 2 was $42 \%$ in the placebo group compared to $25 \%$ in the treatment group. SOD is well tolerated with no serious adverse effects; as such further investigation for ROP prevention is worthwhile. Nuclear factor erythroid 2 (NFE2)-related factor 2 (Nrf2) is a transcription factor which serves multitude functions. Recently its role in reducing oxidative stress (OS) has come under scrutiny. It holds great promise in reducing OS in the premature population. (2-4)

\section{"Because they are essential in the regulation of several cellular processes and serve as signaling molecules for several biological processes, simply eliminating ROS is not an option. (2)"}

Because they are essential in the regulation of several cellular processes and serve as signaling molecules for several biological processes, simply eliminating ROS is not an option. (2)

OS could be described as a gift that keeps on giving. From a pathophysiological perspective, many diseases of prematurity likely represent a convergence between injury and ROS induced alteration in development. These may result in increased susceptibility to chronic diseases in adulthood and possibly more rapid aging $^{2}$ (excessive ROS can shorten telomeres). (5) Indeed, metanalysis shows adults born pre-term are at higher risk of hypertension, myocardial infarction, stroke, type I and II diabetes, metabolic syndrome, and lung disorders. (5)

Mitochondria have been known as the cell's powerhouse since before I studied biology in high school in the early 1970s. More recently mitochondrial dysfunction has been implicated in the development of lung disease. Both the lungs and brain of the premature infant are immature both structurally and functionally. While developmental pathways are different between brain and lung tissue, both require energy. This depends on proper mitochondrial function.

$90 \%$ of ATP production occurs in the mitochondria ${ }^{2}$. This process results in the production of mitochondrial reactive ROS. Damaged mitochondrial DNA has been implicated in the aging process as well as several neurodegenerative diseases. (6) It stands to reason that the lack of antioxidants in the premature infant results in the presence of more mitochondrial reactive ROS, potentiating the damage of mitochondrial DNA. The resulting mitochondrial dysfunction reduces the amount of energy produced, which in turn may result in abnormal development. Recently, endothelial mitochondrial function in human cells isolated from umbilical cord blood has been shown to strongly predict the risk of poor pulmonary outcomes. (2)

Pulmonary parenchyma and vasculature can also be damaged directly by oxygen toxicity. ROS production can also increase with the administration of inhaled NO (iNO) and, conversely, result in further pulmonary vasoconstriction. (2)

What about $\mathrm{FiO}_{2}$ ? At what point does $\mathrm{FiO}_{2}$ result in lung damage? Delivering greater than $60 \%$ oxygen to an adult for greater than 24 hours at normal barometric pressure results in lung injury ${ }^{7}$. Higher pressures associated with mechanical ventilation can only exacerbate this. But what about neonates, particularly the extremely premature? Many clinicians (and studies) use and $\mathrm{FiO}_{2}$ of 0.6 as the upper limit of acceptability or as part of failure criteria in non-invasive ventilation. Others may use 0.5 or 0.4 , but where do these numbers come from?

When neonatology was in its infancy (no pun intended!) an infant of 32-34 weeks gestation was considered very premature and at high risk of demise. Supplemental oxygen and CPAP were the only tools of the day; there was no choice but to accept whatever level of oxygen a baby required to maintain sufficient $\mathrm{PaO}_{2}$, whatever the consequences, even if that meant eventual death. Today we have pushed the limit of viability down to 22 weeks gestational age (GA), and we have sophisticated ventilators and surfactant at our disposal. Obviously, the difference in the state of physiological development between an infant of 22 weeks GA and one of 34 weeks GA are incomparable.

\section{"The field of neonatology today is completely different than it was 60 years ago and yet when it comes to FiO little has changed. If anything, with the push to early extubations and avoidance of intubation at all costs, our tolerance of high oxygen levels has increased."}

The field of neonatology today is completely different than it was 60 years ago and yet when it comes to $\mathrm{FiO}_{2}$ little has changed. If anything, with the push to early extubations and avoidance of intubation at all costs, our tolerance of high oxygen levels has increased. At what point does the cost of OS exceed the cost of intubation and mechanical ventilation, especially when using proven lung protective strategies?

There is very little information regarding the development of antioxidant defense in the premature infant after birth. Markers of OS are higher in preterm infants 3 days after birth than their term counterparts, as well as vitamin E and SOD. Similarly, oxidative protein products and hydroperoxide levels in plasma are higher 7 days post birth, and urine 8-hydroxy-2-deoxy guanosine (8OHdG) levels may be elevated for up to 100 days post birth compared to term infants. (5) Antioxidant mechanisms do improve with postnatal age. For example, blood vitamin $A$ and $E$ levels are higher in preterm infants at discharge than at birth, and SOD levels are higher at 40 weeks corrected than at preterm birth ${ }^{5}$. How these levels compare to normal term infants was not stated. Of note is a study that showed elevated lipid peroxidation was higher in adolescents (13-15 years of age) born preterm independent of history of CLD relative to those born at term.

Oxygen influences cell division, growth and structure. Lung tissue cultured in $80 \% \mathrm{O}_{2}$ c.f. $20 \% \mathrm{O}_{2}$ show a marked decrease in viability, more cell death and lower ATP content. Lower ATP content indicates mitochondrial impairment which reduces the ability of cells to grow. Severe mitochondrial impairment results in cell necrosis rather than apoptosis since apoptosis requires energy to occur: decreased apoptosis is a hallmark of CLD. In addition, alveolar macrophages were abundant in cells cultured at $20 \%$ but not in $80 \%$. (8)

This brings us back to $\mathrm{FiO}_{2}$. 10-week-old rats exposed to varying $\mathrm{O}_{2}$ concentrations showed no difference in levels of derivatives of reactive oxygen metabolites (DROMS) after 24 hours of expo- 
sure to $20.9 \%$ or $35.5 \% \mathrm{O}_{2}$, but they increased at $39.8 \%, 62.5 \%$ and $82.2 \% \mathrm{O}_{2}$ over the same time period, the highest levels being reached at $82.2 \% \mathrm{O}_{2}$. Morphological changes in red blood cells were also observed at the latter levels. Levels of biochemical antioxidant potential did not increase with increasing $\mathrm{O}_{2}$ concentration indicating no adaptation. The authors concluded that exposure to $\mathrm{FiO}_{2}$ above 0.40 for 24 hours resulted in excessive levels of oxidative stress. (9) It is important to note these rats were adults, not preterm pups. As well, the antioxidant systems in rats are somewhat different than in humans; for instance, rats produce their own vitamin C.

Given that $\mathrm{O}_{2}$ concentrations above $40 \%$ tax adult antioxidant capacity, it stands to reason that the premature infant's antioxidant capacity is likely overwhelmed at lower concentrations. Regardless, an $\mathrm{FiO}_{2}$ of 0.40 remains an acceptable parameter in the NICU. Furthermore, the lower the post-partum age the more easily these systems are swamped since they have not yet fully developed. If we are to follow the precautionary principle, we should be exposing our premature patients to the lowest possible $\mathrm{O}_{2}$ concentrations, particularly during the first week of life, and this should most certainly be lower than $40 \%$ if at all possible. I submit that less is better, and the best, safest $\mathrm{FiO}_{2}$ is 0.21 . It is the general practice in the unit in which I work to give surfactant (either minimally invasive or INSURE) to any infant on non-invasive support who requires $30 \% \mathrm{O}_{2}$ or more.

"Given that 02 concentrations above $40 \%$ tax adult antioxidant capacity, it stands to reason that the premature infant's antioxidant capacity is likely overwhelmed at lower concentrations. Regardless, an FiO2 of 0.40 remains an
acceptable parameter in the NICU."

Even after the endogenous antioxidant system is fully functional the consequences of OS remain. Studies have shown that despite increased use of non-invasive modes of ventilation the duration of respiratory support and supplemental $\mathrm{O}_{2}$ have increased, and pulmonary function (PF) at 8 years of age was worse in a cohort studied from 2005 compared to an earlier cohort from 1997. While all former preterm children had PF impairment relative to their term counterparts, the degree of impairment increased with history of CLD or requirement of supplemental $\mathrm{O}_{2}$ at 36 weeks corrected age. (10) This supports the surmission that prolonged exposure to supplemental $\mathrm{O}_{2}$ results in greater airway smooth muscle proliferation leading to reactive airway disease.

What are the implications for respiratory support? The benefits of an "open lung" approach to respiratory support are well known but at the bedside all too often what represents an "open lung" is misunderstood. Too many clinicians are still fixated on "rib counts" on a chest film without considering the quality of the lung fields; are they hazy or patchy or well aerated? Optimum PEEP coincides with optimum compliance. At optimum PEEP several factors interact favourably, such as compliance, cardiac output, and tissue oxygenation. (11) Simply put, when PEEP is optimum, ventilating pressures and $\mathrm{FiO}_{2}$ are lowest and $\mathrm{SpO}_{2}$ is highest at a given $\mathrm{FiO}_{2}$. If $\mathrm{FiO}_{2}$ is low and blood pressure is acceptable the patient is likely physiologically not hyperinflated, regardless of what a chest film might show. Ventilation mode also must be considered, for what is considered "hyperinflated" in a conventional mode of ventilation may be perfectly acceptable in a high frequency mode since large tidal breaths do not contribute to sheer stress. Simply put, when mechanically ventilating a premature infant, one should aim for settings that minimize $\mathrm{FiO}_{2}$. This includes non-invasive ventilation (NIV).

Finally, there are many other factors that increase the production of ROS and resulting OS. While growth and development require significant energy, the premature infant's limited ability to feed and absorb nutrients from their immature gut necessitates supplementing nutrition parenterally. As previously mentioned, TPN preparations contain ROS. Breast milk is well known to be the best food for babies and its benefits are particularly pronounced in the preterm population. Formula, and fortifiers it contains, increase OS compared to breast milk. Blood transfusions increase the production of ROS due to increased iron availability, and phototherapy increases the production of ROS. OS is also greater in infants who are small for gestational age. Inflammation secondary to infection is another contributor to the formation of ROS and $\mathrm{OS}^{5}$. While our focus on OS has been on pulmonary development, OS also affects brain development. Readers interested in OS effects on neurodevelopment are directed to reference 5.

In conclusion, it is time clinicians reevaluate the use of $\mathrm{O}_{2}$ in the premature population with an eye to reducing $O S$ and its sequelae. This includes lowering our tolerance for high $\mathrm{FiO}_{2}$ in NIV.

Our patients deserve nothing less.

\section{References:}

1. https://pubmed.ncbi.nlm.nih.gov/3144189/

2. https://www.ncbi.n/m.nih.gov/pmc/articles/PMC6662609/

3. https://pubmed.ncbi.n/m.nih.gov/2264459/\#: : :text=A\%20 series $\% 20$ of $\% 20$ conditions $\% 20$ in. Oxygen $\% 2$ radical $\% 20$ disease $\% 20 \mathrm{in} \% 20$ neonatology $\% 22$.

4. $\quad$ https://www.ncbi.n/m.nih.gov/pmc/articles/PMC4680839/

5. https://www.sciencedirect.com/science/article/piil S2213231717309849

6. $\quad$ https://www.ncbi.n/m.nih.gov/pmc/articles/PMC6942696/

7. https://www.hindawi.com/journals/nrp/2011/260482/

8. https://www.nature.com/articles/s41598-019-52813-2

9. https://www.hindawi.com/journals/omcl/2012/381763/

10. https://www.resmedjournal.com/artic/e/S09546111(18)30033-7/pdf

11. Scanlan, C.L., Heuer, A.J., Sinopoli, L.M., Certified Respiratory Therapist Exam Review Guide, Jones \& Bartlett, 2010: page 353. (https://books.google.ca/books?id=/4ZZog8rWSUC\&pg =PA353\&lpg=PA353\&dq=pulmonar $y+$ optimum + compliance + definition \& source $=b / \&$ ots $=k K$ D6jnyaTo\&sig=ACfU3U2i6SiEtLwhYHHEoHjgGjyr7xoj$\underline{m g \& h l=e n \& s a=X \& v e d=2 a h U K E w j J n f K} p 83 x A h W H Z s O K H$ ZvaAacQ6AF6BAgYEAM\#v=onepage\&q=pulmonary $\% 20$ optimum\%20compliance\%20definition\&f=false)

Disclosures: The author receives compensation from Bunnell Inc for teaching and training users of the LifePulse HFJV in Canada. He is not involved in sales or marketing of the device nor does he receive more than per diem compensation. Also, while the author practices within Sunnybrook H.S.C. this paper should not be construed as Sunnybrook policy per se. This article contains elements considered "off label" as well as maneuvers, which may sometimes be very effective but come with inherent risks. As with any therapy, the riskbenefit ratio must be carefully considered before they are initiated.

\section{NT}




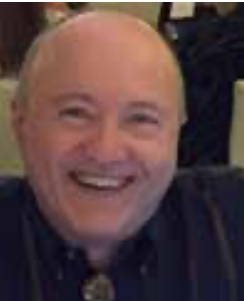

Rob Graham, R.R.T./N.R.C.P.

Advanced Practice Neonatal RRT

Sunnybrook Health Science Centre

43 Wellesley St. East

Toronto, ON

Canada M4Y 1H1

Email: rcanrcp57@yahoo.ca

Telephone: 416-967-8500
New subscribers are always welcome! NEONATOLOGY TQDAY

To sign up for free monthly subscription, just click on this box to go directly to our subscription page
Oo National Perinatal Association PERINATAL MENTAL HEALTH

nationalperinatal.org/position

www.nationalperinatal.org/mental_health

\section{EDUCATE PROVIDERS}

All perinatal health care providers need training and education that

will help them support families.

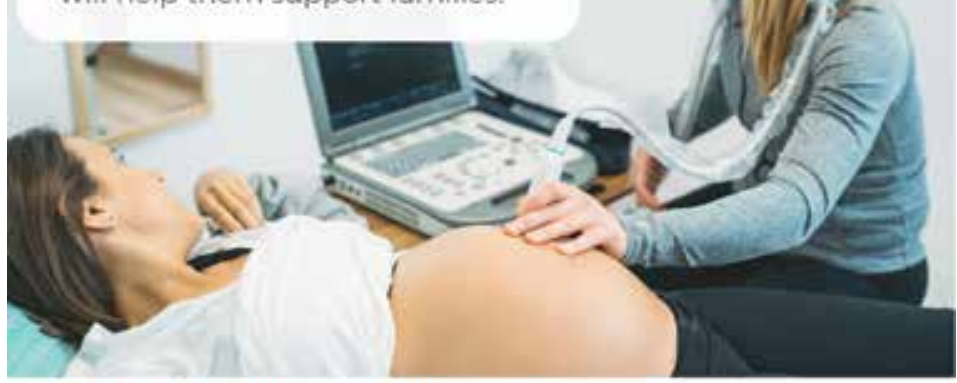

Educate. Advocate. Integrate.

\section{Readers can also follow NEONATOLOGY TO}

\section{via our Twitter Feed \\ @NEOTODAY}

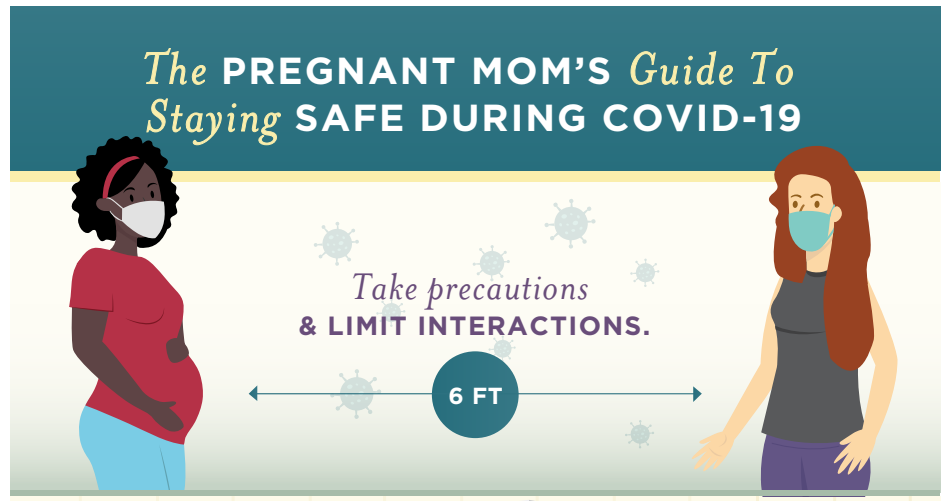

Maintain at least

A 30-DAY SUPPLY

OF YOUR MEDICATIONS.
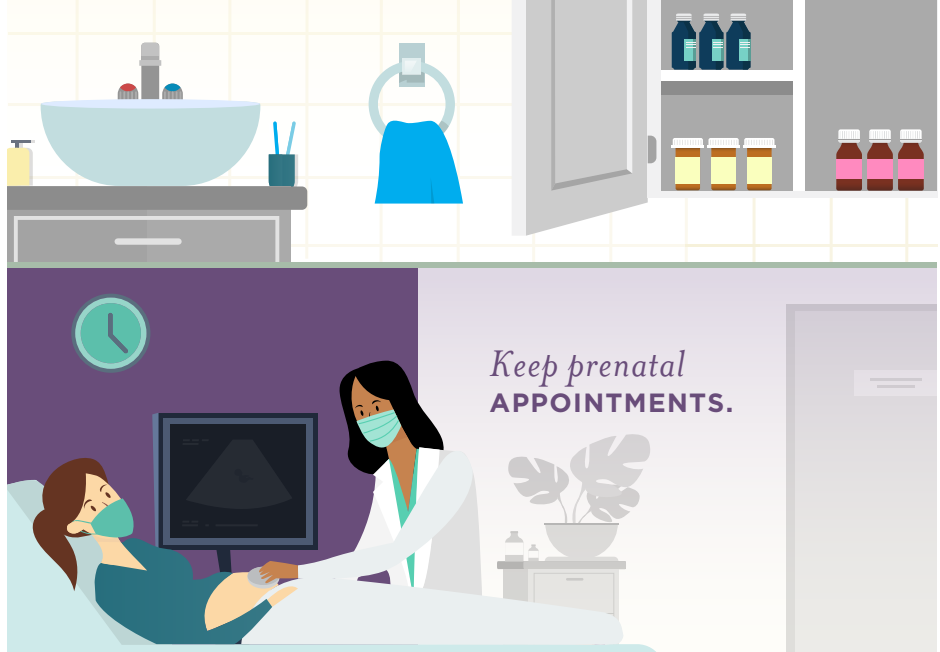

Keep prenatal APPOINTMENTS.

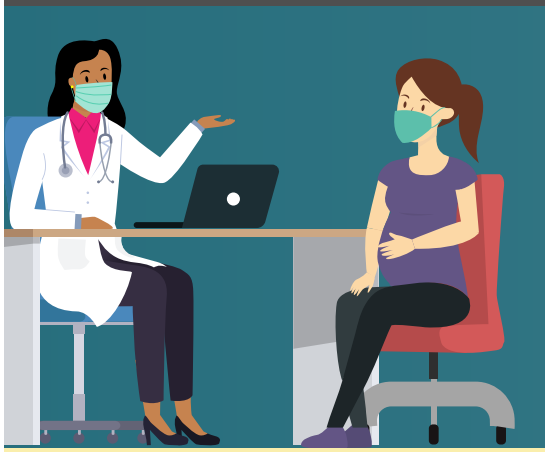

Talk to your health care provider about STAYING SAFE DURING COVID-19.

LEARN MORE

$\mathrm{NCfIH}$ 